\title{
Commensurate vortex lattices and oscillation effects in superconducting Mo/Si and W/Si multilayers
}

\author{
M.Yu. Mikhailov' ${ }^{1}$, O.I. Yuzephovich ${ }^{1}$, Yu.V. Bomze ${ }^{1,2}$, \\ E.I. Buchstab ${ }^{2}$, and N.Ya. Fogel ${ }^{2}$ \\ ${ }^{1}$ B. Verkin Institute for Low Temperature Physics and Engineering \\ of the National Academy of Sciences of Ukraine, 47 Lenin Ave., Kharkov 61103, Ukraine \\ ${ }^{2}$ Solid State Institute, Technion, Haifa, 32100 Israel \\ E-mail: mikhailov@ilt.kharkov.ua
}

Received August 6, 2004

\begin{abstract}
We report experimental results of the vortex lattice structure investigation in the artificial superconducting $\mathrm{Mo} / \mathrm{Si}$ and $\mathrm{W} / \mathrm{Si}$ superlattices. The resistance $R$ and critical current $I_{C}$ measurements in parallel magnetic fields have been performed as well as measurements in tilted magnetic fields. At temperatures where condition of strong layering is satisfied the dependences $I_{C}\left(H_{\|}\right)$and $R\left(H_{\|}\right)$reveal oscillation behavior. It is shown that the appearance of oscillations and of reentrant behavior (vanishing of resistivity in definite ranges of $H_{\|}$) are due to the strong intrinsic pinning and to the effect of commensurability between the vortex lattice period and multilayer wavelength. The locations of $I_{C}\left(H_{\|}\right)$and $R\left(H_{\|}\right)$extrema correspond to the stable states of a commensurate vortex lattice. Our experimental data are in good quantitative agreement with Ivlev, Kopnin, and Pokrovsky (IKP) theory. It is shown that the values of the commensurability fields depend exclusively on the superlattice period $s$ and anisotropy coefficient $\gamma$ and do not depend on the type of materials used for multilayer preparation. The memory effect, i.e., dependence of the oscillation pattern on the magnetic history of the sample, is observed. It is shown experimentally that the state of the vortex matter in the layered superconductors is essentially different from that of type-II superconductors with a random distribution of the pinning centers. Investigation of oscillation and reentrance behavior may be used as a new tool for vortex lattice arrangement study in layered superconductors. The essential advantage of this method is connected with its simplicity and with the possibility of using it in arbitrary large fields. Investigations of the commensurate states may be used for rather precise determination of the anisotropy coefficient $\gamma$.
\end{abstract}

PACS: 74.78.Fk, 74.25.Qt

\section{Introduction}

The vortex lattice (VL) structure in layered superconductors differs in many aspects from the simple triangular one observed in homogeneous type-II superconductors, unless the magnetic field $\mathbf{H}$ is parallel to the $\mathbf{c}$ axis of the system considered. Different types arrangements of VL are predicted theoretically for the cases when the applied magnetic field is parallel or inclined with respect to the layer planes. The first theory concerning VL structure in the layered superconducting systems consisting of alloys with periodic modulation of the component concentration appeared many years ago [1]. It was shown that the spatial vari- ation of the impurity concentration might be considered as a regular pinning potential. Ami and Maki [1] have studied the stability of different VL configurations in the Ginzburg-Landau (GL) approximation for parallel magnetic field $H_{\|}$taking into account that the «resonance conditions», i.e., the commensurability between vortex lattice parameter and layered structure period, plays an important role. In the vicinity of the upper critical field $H_{c 2}$ in the commensurate state the triangular VL, with different orientations of the vortex unit cell vector with respect to the layers, appeared to be stable. The values of magnetic fields $H_{\Delta}$ corresponding to the resonance conditions have been obtained. In the framework of this theory they 
depend exclusively on the superlattice period $s$. At these fields the maxima of the critical current $I_{c}$ should be observed. It appeared that the Ami and Maki model described fairly well the dependence of $I_{c}$ on $H_{\|}$obtained on the modulated PbBi alloys [2].

Interest in the commensurability effect and in the properties of the VL structure has been renewed after discovery of the high-temperature superconductivity in the layered oxide compounds. Especially essential progress was achieved in the area of the vortex lattice structure theory. Different theoretical approaches including anisotropic three-dimensional (3D) London and GL models as well as the Lawrence-Doniach (LD) model, were used. According to the results of Ref. 3 obtained within the London approximation in the field domain $H_{c 1} \ll H<<H_{c 2}$ the unit cell of the flux line lattice should be strongly distorted with respect to the equilateral triangle. Choosing the primitive cell of the VL as a hexagon, Campbell, Doria, and Kogan [3] obtained the unit cell parameters of $\mathrm{VL}$, which depend intrinsically on the anisotropy parameter $\gamma=(\mathrm{M} / \mathrm{m})^{1 / 2}$ and the angle between $\mathbf{H}$ and the anisotropy axis. Here $M$ is the effective electron mass along the $\mathbf{c}$ axis, and $m$ is the inplane mass. The commensurability effect has been outside the scope of this work. The latter was considered in the papers of Ivlev, Kopnin, and Pokrovsky [4-6]. It was shown [4] that in the case of parallel field, when the intrinsic pinning energy $E_{p}$ is much greater than the elastic energy of a VL shear deformation $E_{\mathrm{el}}$, the vortices cannot cross the layers. In this situation the period $Z_{0}$ along the $\mathbf{c}$ axis is fixed, and it is determined by the initial conditions under which the VL was formed. This means that the VL should always be commensurable with the layered structure period $s$ (the distance between vortices in the direction orthogonal to the layers $Z_{0}=N s, N$ is an integer). It is independent of the external field, while the unit cell area varies with a field only due to the vortex displacements along the layers. In the opposite limiting case the VL parameters are determined by the external magnetic field. It was shown that the free energy of the rhombic lattice in the commensurate state as a function of $H$ displays two minima corresponding to the different orientations of the unit cell vectors with respect to the layer planes [4]. There are a lot of metastable states in the instability region of the rhombic lattice corresponding to the different displacements of the vortex rows relative to each other in the neighboring interlayers [4,7]. The number of such states grows at low magnetic fields. They can be dynamically accessible upon $H$ variation [7]. In the framework of the LD approach for relatively high parallel magnetic fields a sequence of first-order phase transitions between VLs with different $N$ is predicted [8]. The range of the stability of the VL struc- tures with the different orders of commensurability $N$ was investigated theoretically in Ref. 9.

For the tilted magnetic fields many types of the vortex arrangement are suggested [10-19]. The idea of an independent response of the layered superconductor to the parallel and perpendicular components of magnetic field [20] is exploited in many works. The most exotic among possible VL configurations in the tilted magnetic fields is the so-called combined lattice consisting of two vortex «species» oriented in different directions (see, for example, Refs. 10-15, 18, 19, $21)$. Such a configuration of VL may appear only in a case of a rather large $\gamma[10,12]$. One of the coexisting sublattices is oriented along $\mathbf{c}$ axis; another may be parallel to the layers [13] or oriented along the external field [11]. In the vicinity of $H_{c 1}$ for the tilted magnetic fields the vortex configuration consisting of flux-line chains is predicted [17]. Such vortex chains in the weak field range have been observed in decoration experiments on high- $T_{C}$ superconductors [22-24].

Obviously, due to the nonstandard VL structure and intrinsic pinning, many unusual effects can arise in layered superconductors. First of all, in a tilted field the direction of the magnetization vector $\mathbf{M}$ in many cases does not coincide with the external field direction $[3,16,25]$. Depending on the misorientation angle, anisotropy parameter and magnetic field domain, the vector $\mathbf{M}$ may be oriented either closely to the $\mathbf{c}$ direction of the layered structure [3], along the layer planes $[11,25]$, or along the applied field direction [11]. Even magnetization $\mathbf{M}$ antiparallel to the external field $\mathbf{H}$ is possible [3,26]. In parallel magnetic fields due to the intrinsic pinning and commensurability effect the critical current (when the transport current flows in the layer planes) should be an oscillating function of magnetic field $[1,4]$. This result was obtained experimentally, as was mentioned above, on alloys with a spatial modulation of the component concentration [2] and on $\mathrm{Nb} / \mathrm{Ta}$ multilayers [27]. The theoretical results cited above have been obtained mostly for high- $T_{C}$ superconductors. These results should be relevant for the artificial multilayers as well. The essential advantage of the artificial superlattices for the VL structure study is their tunability, i.e., the possibility of easily changing their periodicity and anisotropy parameter. Moreover, the present-day technology of their preparation is very sophisticated, and exceptionally perfect superstructures may be obtained.

Here we report on a new effect observed on $\mathrm{Mo} / \mathrm{Si}$ and $\mathrm{W} / \mathrm{Si}$ artificial superlattices, whose origin may be connected with the intrinsic pinning, the commensurability phenomenon, and the specifics of the VL structure in layered superconductors. In a parallel mag- 
netic field and in tilted fields at relatively small tilting angles with respect to the layer planes the reentrance to the state with zero resistivity with increase of the magnetic field is observed. The reentrance phenomenon is detected by the resistive method. The resistivity vs magnetic field $H$ curves below a definite temperature $T_{0}$ become nonmonotonic. At some value of the external magnetic field a resistance minimum appears which becomes more pronounced with decreasing temperature and transforms into a large zero-resistance region (ZRR) at still lower temperatures. After the ZRR the resistance appears again. These effects are very sensitive to the magnetic field orientation. It is shown that all the features of the reentrance behavior may be explained quantitatively if one takes into account different possible realizations of VL structure in layered superconductors. It is suggested that the reentrance phenomenon discovered could be used as a new powerful instrument for the VL structure investigations, which, in distinction from other known methods, is valid for arbitrary high fields. A brief report about some of the results described below for Mo/Si SLs has been published in Ref. 28.

\section{Sample preparation and experimental methods}

For the experiments the $\mathrm{Mo} / \mathrm{Si}$ and $\mathrm{W} / \mathrm{Si}$ multilayered samples where only spacer thickness and, correspondingly, anisotropy parameter is varied have been chosen (Table). At such a choice mainly the parameter $\gamma$ changes, and it allows one to study evolution of the reentrant and oscillation resistive behavior with $\gamma$ variation. The interlayer coupling energy changes with the spacer thickness exponentially [29], and the anisotropy changes quickly as well.

Table. Parameters of multilayered samples

\begin{tabular}{|c|c|c|c|c|c|c|c|}
\hline $\begin{array}{c}\text { Sam- } \\
\text { ple }\end{array}$ & $\begin{array}{l}\text { Multi- } \\
\text { layer } \\
\text { type }\end{array}$ & $\begin{array}{c}\text { Mo } \\
\text { layer } \\
\text { thick- } \\
\text { ness } \\
d_{\mathrm{Mo}}, \AA \\
\end{array}$ & $\begin{array}{c}\mathrm{W} \\
\text { layer } \\
\text { thick- } \\
\text { ness } \\
d_{W}, \AA \\
\end{array}$ & $\begin{array}{c}\text { Si layer } \\
\text { thickness } \\
d_{\mathrm{Si}}, \AA\end{array}$ & $\begin{array}{c}\text { Number } \\
\text { of } \\
\text { bilayers }\end{array}$ & $\gamma$ & $T_{C}, \mathrm{~K}$ \\
\hline A & $\mathrm{Mo} / \mathrm{Si}$ & 22 & & 28 & 50 & 5.7 & 4.17 \\
\hline B & $\mathrm{Mo} / \mathrm{Si}$ & 22 & & 34 & 50 & 11.8 & 3.67 \\
\hline $\mathrm{C}$ & $\mathrm{Mo} / \mathrm{Si}$ & 22 & & 44 & 50 & 23.5 & 4.15 \\
\hline D & $\mathrm{W} / \mathrm{Si}$ & & 20 & 30 & 10 & 3.2 & 3.32 \\
\hline E & $\mathrm{W} / \mathrm{Si}$ & & 20 & 40 & 10 & 15.2 & 2.92 \\
\hline
\end{tabular}

The Mo/Si multilayers were prepared by dc magnetron sputtering in argon onto glass substrates. The $\mathrm{W} / \mathrm{Si}$ multilayers were prepared by $e$-beam evaporation onto oxidized Si (100) wafers. More details may be found in Refs. 29-31. The sample wavelength and individual layer thicknesses are determined from small angle x-ray diffractometry with the accuracy $0.1 \AA$. Si layers in multilayers are amorphous. The metal layers have fine crystalline structure.

Measurements were performed with 5T superconducting magnet. At all orientations of $\mathbf{H}$ with respect to the layer planes the transport current I directed along the layers was always perpendicular to $\mathbf{H}$. The precision of $\mathbf{H}$ alignment with the layer planes was about $0.2^{\circ}$. The stabilization of the temperature during a field sweep was no worse than $10^{-3} \mathrm{~K}$. The zero-field resistive transition width for the all samples was no more than $0.15 \mathrm{~K}$.

The values of the anisotropy parameter $\gamma$ were obtained from measurements of the critical magnetic fields $H_{c||}(T)$ and $H_{c \perp}(T)$.

\section{Experimental results and discussion}

In Fig. 1 the resistance as a function of magnetic field at different temperatures for angle $\theta=0^{\circ}$ is shown for

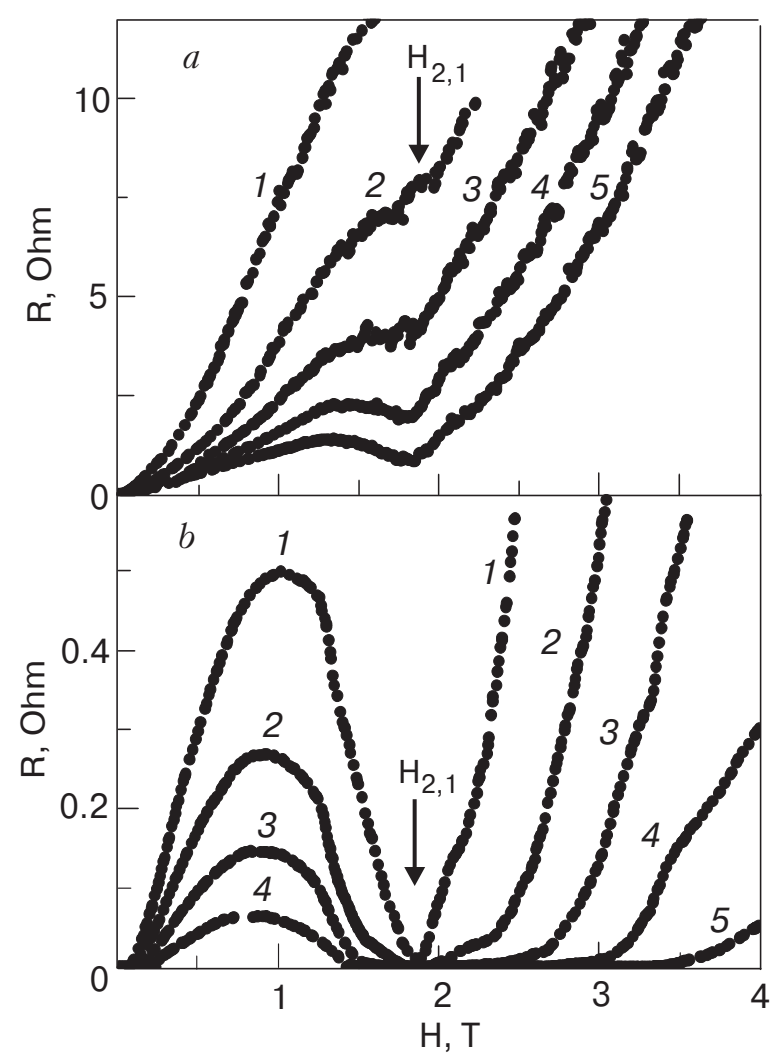

Fig. 1. Resistance as a function of magnetic field for $\mathrm{Mo} / \mathrm{Si}$ sample $\mathrm{B}$ (angle $\theta=0^{\circ}$ ) at different temperatures $T, \mathrm{~K}$ : (a) 3.557 (1); 3.535 (2); 3.520 (3); 3.508 (4); 3.495 (5); (b) 3.456 (1); 3.420 (2); 3.377 (3); 3.314 (4); 3.214 (5). 


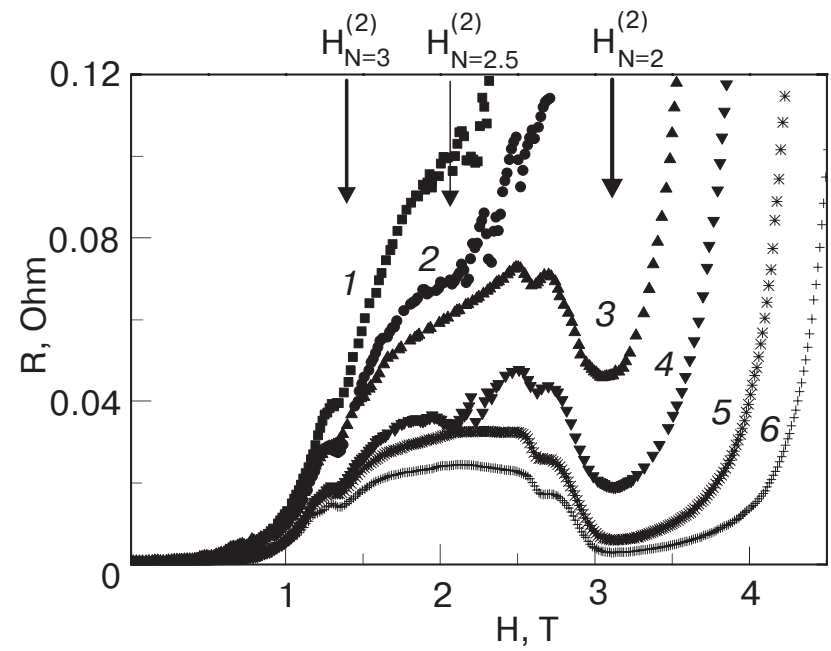

Fig. 2. Resistance as a function of parallel magnetic field for Mo/Si sample A at different temperatures $T$, K: 3.788 (1); 3.779 (2); 3.765 (3); 3.750 (4); 3.731 (5); 3.711 (6).

$\mathrm{Mo} / \mathrm{Si}$ sample B with the wavelength $56 \AA\left(\theta=0^{\circ}\right.$ for magnetic field parallel to the layers). Starting at some reduced temperature ( $t=0.96$ in this case) steps appear on the $R$ vs $H$ curves, which at lower temperatures develop into minima. With further decrease of $T$ instead of resistance minima, zero-resistance regions (ZRR) appear (Fig. 1,b), which manifest the reentrance of superconductivity with the applied field increase. After the ZRR at still larger fields the resistance appears again. As we keep going down in temperature, the ZRR becomes wider, and in the low temperature limit, the $R$ maximum dividing two minima vanishes (plot 5, Fig. 1,b). Thus, reentrance behavior exists in a limited temperature range between $T_{0}$ and some lower temperature $T^{\prime}$. For understanding the reentrance phenomenon scale it should be mentioned that the low-temperature normal resistance of sample B is $42.4 \mathrm{Ohm}$.

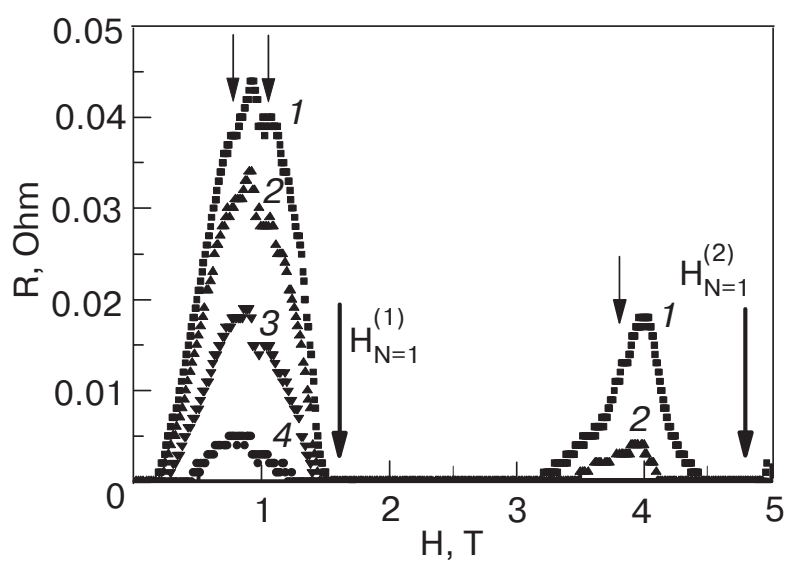

Fig. 3. Resistance as a function of magnetic field for $\mathrm{Mo} / \mathrm{Si}$ sample B (another realization) at different temperatures $T$, K: 3.236 (1); 3.218 (2); 3.172 (3); 3.077 (4).
The resistance as a function of parallel magnetic field for $\mathrm{Mo} / \mathrm{Si}$ sample A with a wavelength $50 \AA$ is shown in Fig. 2. A similar plot for sample C $(s=66 \AA)$ has been presented in Ref. 28. For all samples the magnetic field values corresponding to the ZRRs and to the resistance minima are different.

It is noteworthy that different patterns of $R$ vs $H_{\|}$ curves may be obtained on the same sample. The reentrance phenomenon is sensitive to the magnetic history. For example, when one starts measurements with the first switching on of the magnetic field sweep at sufficiently low temperature, another realization of the $R$ vs $H$ curves at $\theta=0^{\circ}$ in the same field range may be obtained. Such another realization for sample B is shown in Fig. 3. As follows from this figure, the reentrance of superconductivity may arise twice during one magnetic field sweep. The third realization for this sample is shown in Fig. 4. None of the patterns observed on sample B coincides with those registered for other samples.

The features of the resistive curves in a parallel magnetic field depend essentially on the transport current (Fig. 5). At relatively small currents (plot 1 in Fig. 5) the resistive state first appears at rather large magnetic field. Until this field the Lorentz force is too small for overcoming of the pinning barrier. At a

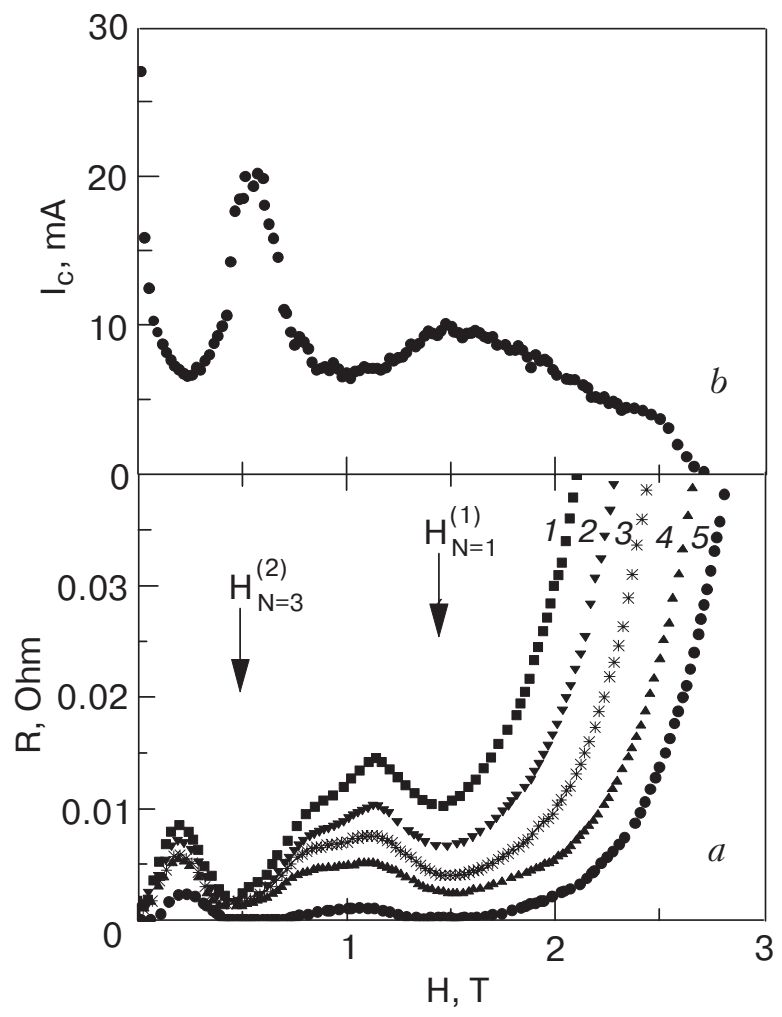

Fig. 4. $a$ - Resistance as a function of magnetic field for $\mathrm{Mo} / \mathrm{Si}$ sample B (the third realization) at different temperatures $T$, K: 3.462 (1); 3.459 (2); 3.453 (3); 3.447 (4); $3.441(5) . b-$ Critical current as a function of magnetic field for this sample at $T=3.4 \mathrm{~K}$. 


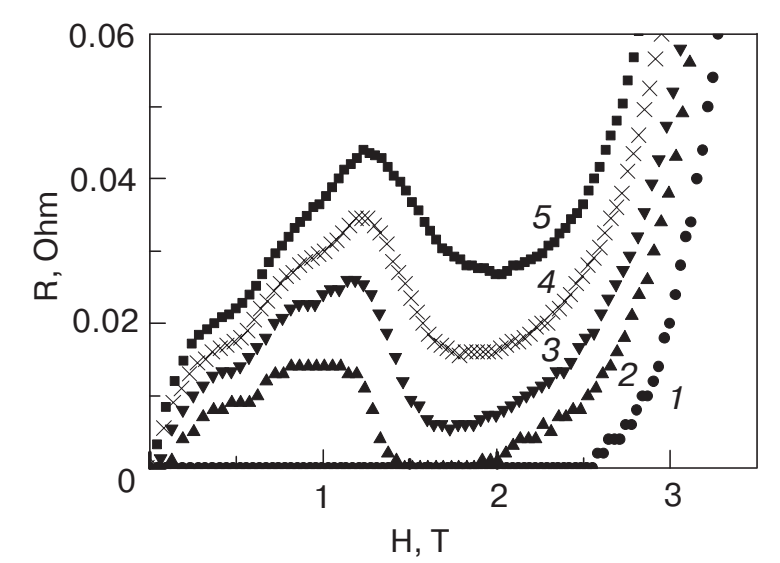

Fig. 5. Resistance as a function of magnetic field for $\mathrm{Mo} / \mathrm{Si}$ sample $\mathrm{B}$ at $T=3.37 \mathrm{~K}$ for different transport currents: 0.5 (1); 1.0 (2); 1.5 (3); 2.0 (4); 2.5 (5) mA.

higher current (plot 2) the resistance is already observed in the weak field range, but at intermediate fields the reentrance of superconductivity is observed. Further current increase leads to the appearing of a resistance minimum (plot 3) instead of the ZRR. Thus the correct choice of the transport current value is crucial for the observation of the ZRR and nonmonotonic $R$ vs $H_{\|}$dependence behavior. As we will see below, the most informative are the resistive curves of the plot 3 kind.

From comparison of Fig. 4 and 5 it follows that diminishing of temperature and diminishing of the transport current give rise to similar evolution of the resistive transitions. In the former case the pinning barrier increases, while in the latter case the Lorentz force decreases. Because the Lorentz force should counterbalance intrinsic pinning for the vortex motion, it is obvious that both factors influence the resistive behavior identically. If one wants to investigate the larger part of the $H_{\|}-T$ phase diagram, it is necessary to enhance the transport current value at the reduced temperature. The temperature $T^{\prime}$ appears to be a function of transport current, and this temperature may be set as low as one wishes.

In the temperature range between $T_{0}$ and $T^{\prime}$ hysteretic behavior was observed. Some examples of hysteretic curves are shown in Fig. 6. Though the resistance in the increasing and decreasing magnetic fields differs markedly, the positions of the extrema, as a rule, coincide. Similar hysteretic behavior is observed for all samples and for all detected realizations.

The $R$ vs $H_{\|}$dependences at different temperatures for $\mathrm{W} / \mathrm{Si}$ sample D $(s=50 \AA)$ are shown in Fig. 7. The resistive curve patterns obtained on this and other $\mathrm{W} / \mathrm{Si} \mathrm{SLs}$ are similar to those obtained on $\mathrm{Mo} / \mathrm{Si}$ samples. In the resistive transition realization presented in Fig. 7, the resistance minima and ZRRs appear at three different fields. On $\mathrm{W} / \mathrm{Si}$ multilayers
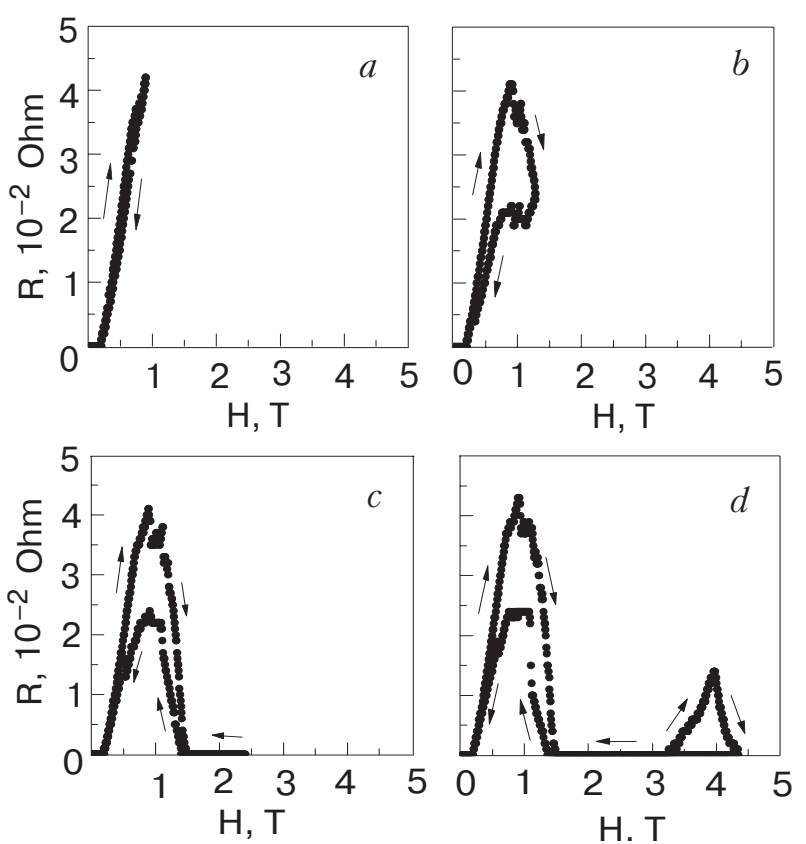

Fig. 6. Hysteretic behavior of resistance for $\mathrm{Mo} / \mathrm{Si}$ sample B at temperature $T=3.236 \mathrm{~K}$.

the hysteretic behavior and the transport-current dependence of the resistive curve are akin to those observed on Mo/Si SLs.

The reentrance behavior is sensitive to the deviation of magnetic field orientation from parallel to the layer planes. It is illustrated by Fig. 8 where the resistance as a function of magnetic field is shown for different angles $\theta$. When the misorientation angles between $\mathbf{H}$ and the layer planes are very small the resistance increases with angle growth, but all the features of the resistance transition at $\theta=0^{\circ}$ hold, the positions of the $R$ minima do not shift (Fig. 8,a), and the reentrance phenomenon also takes place. It was shown that such a behavior on $\mathrm{Mo} / \mathrm{Si}$ multilayers is

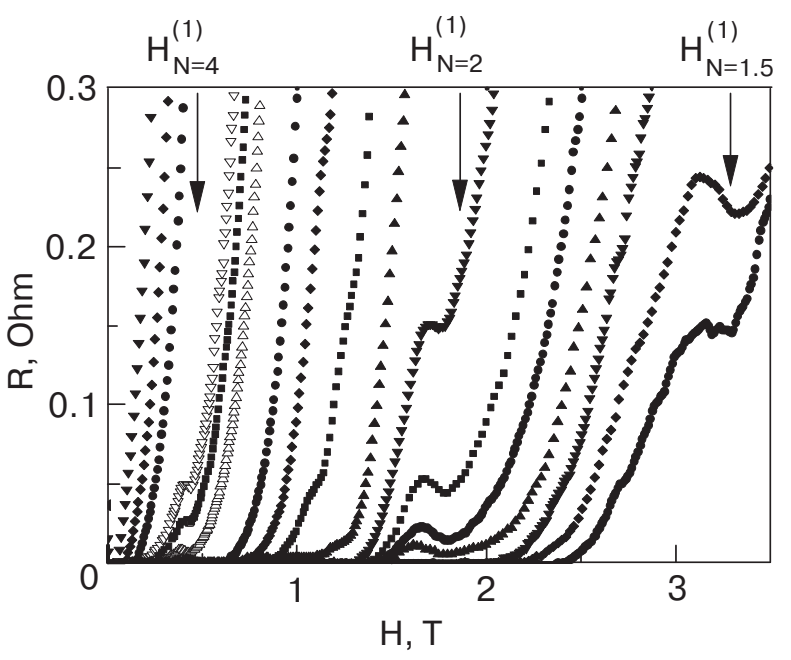

Fig. 7. Resistance as a function of magnetic field at different temperatures for $\mathrm{W} / \mathrm{Si}$ sample $\mathrm{D}$ in the temperature range $2.895-2.488 \mathrm{~K}$. 
an indication of the lock-in transition at small angles $\theta<\theta_{\text {cr }}$ [32]. In this $\theta$ range the vortices are trapped in a position parallel to the layers though the applied magnetic field is tilted with respect to the layers. The $\theta_{\mathrm{cr}}$ is usually defined as the angle where the directions of the external magnetic field and of the induction in the sample begin to coincide. The maximal value of $\theta_{\text {cr }}$ for sample $\mathrm{B}$ is about $2^{\circ}$. At the larger angles in a case of sufficiently low temperatures the minimum position as well as all resistive curves shift to the smaller $H$ side, and this shift increases with growth of $\theta$ (Fig. $8, b)$. At another critical angle $\theta_{\mathrm{cr}}^{*}$ the resistance minimum disappears. Both angles $\theta_{\mathrm{cr}}$ and $\theta_{\mathrm{cr}}^{*}$ depend on the temperature. Most probably, the observation of patterns similar to those typical for commensurate states in a parallel field [28] over a wide angle range may be considered as evidence of the existence of the combined vortex lattice predicted in Refs 10-17. This question will be discussed in detail elsewhere.

As was natural to expect, oscillations of the critical current $I_{c}$ are observed along with the resistivity oscillations (Fig $4, b$ ). The positions of the minima on
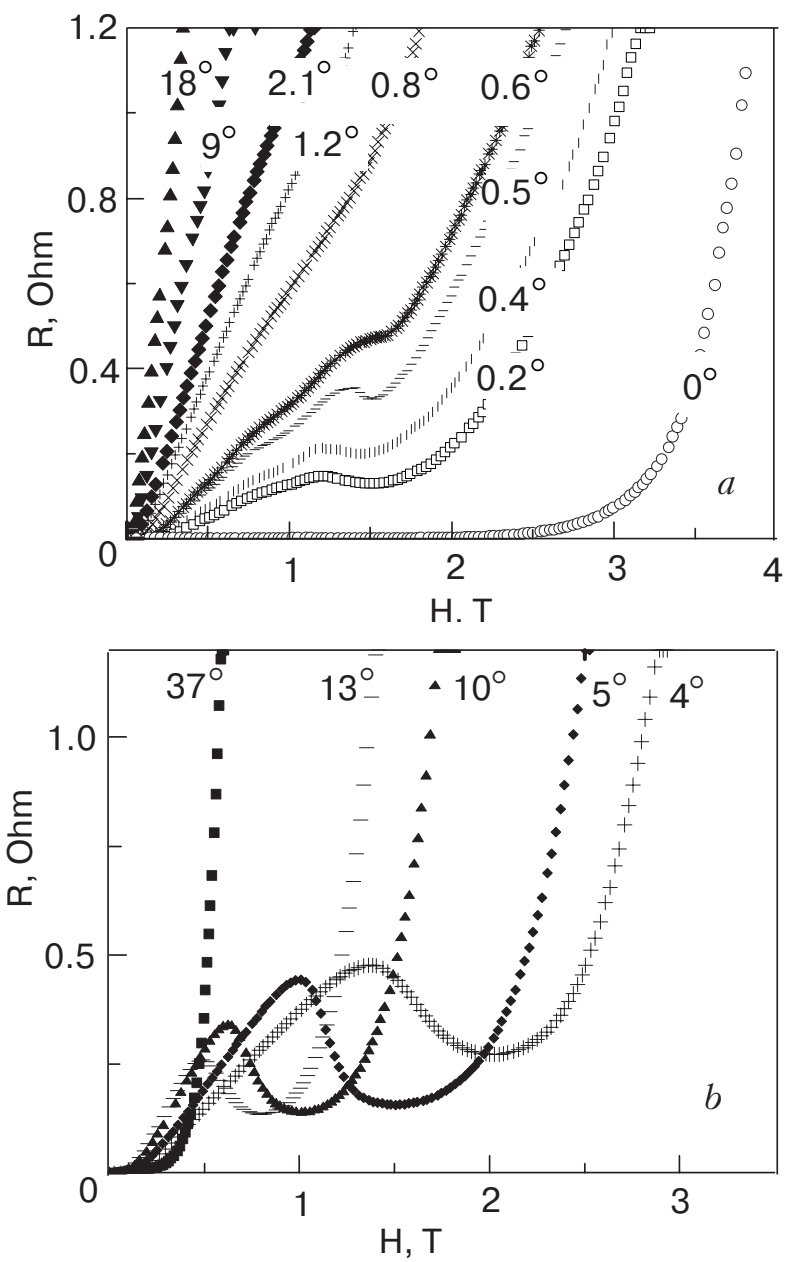

Fig. 8. Resistance as a function of magnetic field for different angles $\theta$ at $T=3.440(a)$ and $3.193 \mathrm{~K}(b)(\mathrm{Mo} / \mathrm{Si}$ sample B). the $R$ vs $H_{\|}$curves coincide with the locations of the maxima on $I_{c}$ vs $H_{\|}$curves and vice versa. The dependences of the critical current on $H_{\|}$display hysteretic behavior, too. Thus the explanation of the unusual patterns characteristic for the $R$ vs $H_{\|}$ dependences is very simple. Provided there are oscillations on the $I_{c}$ vs $H$ curves, one may expect that for the fields around the $I_{C}$ maximum the fixed transport current $I$ appears to be less than $I_{c}$, and correspondingly resistance is absent (ZRR state). In the meantime, in magnetic fields around the $I_{c}$ minimum the condition $I>I_{C}$ may be satisfied, and a nonzero resistance should be observed in this field range. The data in Fig. 5 also serve as the confirmation of explanation presented above.

For a detailed interpretation of the data obtained let us begin with the determination of the physical meaning of the temperature $T_{0}$ where the signs of developing ZRR states appear. It follows from the experimental data that at this temperature the transverse coherence length $\xi_{\perp}(T)$ becomes equal to or less than $s / 2\left(s=d_{\text {met }}+d_{\mathrm{Si}}\right.$ is the SL wavelength), i.e., beginning at this temperature a confinement of the vortices between superconducting layers starts to develop. For sample $B$ the value $\xi_{\|}(0)=63 \AA, \gamma=11.8$, $\xi_{\perp}(0)=5.5 \AA$. At $T_{0}=3.52 \mathrm{~K}$ the value $\xi_{\perp}\left(T_{0}\right)=27 \AA$, $2 \xi_{\perp}\left(T_{0}\right) \leq s=56 \AA$. When the vortex cores fit into the insulating interlayers, intrinsic pinning should become well pronounced [33], and a transition to the limit $E_{p}>E_{\mathrm{el}}$ may be expected. Thus, $T_{0}$ manifests by itself the transition to the regime of strong layering [4]. It should be mentioned that the temperature $T_{0}$ is practically the same as the $3 \mathrm{D}-2 \mathrm{D}$ crossover temperature determined from the temperature dependence of the critical magnetic field $H_{c \|}$. The same situation is observed for other samples. The appearance of nonmonotonic $I_{c}$ vs $H_{\|}$dependence only below the crossover temperature is also reported in Ref. 27.

Based on a suggestion [28] that the phenomena observed are the manifestation of the existence of commensurate vortex lattices, let us analyze the data obtained in detail. In the beginning let us compare the results of different theories based on various approaches. The Ami-Maki theory [1] considers only dirty superconductors, and its domain of validity is restricted to fields close to $H_{c 2}$. It is assumed that the spatial variation of the impurity content is relatively small (on a scale of the electron mean free path), and among all superconductor parameters only the diffusion coefficient has significant spatial dependence on the impurity concentration. For the specific fields $H_{\Delta}$ satisfying the «resonance condition» (i.e., the condition that the Abrikosov lattice parameter $a$ is commen- 
surate with the modulation period $s$ ) the following simple expression is obtained [1]:

$$
H_{\Delta}=\frac{\sqrt{3} \pi \Phi_{0}}{2 s^{2}}\left(N^{2}+L^{2}+N L\right)^{-1}
$$

Here $N$ and $L$ are integers. This solution suggests that not only vortex density variation is possible, but rotation of the vortex unit cell with respect to the equiconcentrational planes as well. Formula (1) satisfactorily describes the experimental data obtained by Raffi et al. [2] on PbBi alloys with periodically modulated concentration. The consistency of the experimental results of Broussard et al. [34] ( $\mathrm{Nb} / \mathrm{Ta}$ artificial multilayers) with the Ami-Maki predictions is essentially worse. It is clear that the Ami-Maki approximation is valid only for a case of weak anisotropy. As our numerical estimates show, there is nothing in common between the calculated $H_{\Delta}$ values and the experimental values of $H_{N}$ of the $I_{c}$ maxima and $R$ minima on the resistive curves for $\mathrm{Mo} / \mathrm{Si}$ and $\mathrm{W} / \mathrm{Si}$ samples investigated.

The serious extension of the Ami-Maki model has appeared much later, after the discovery of high- $T_{c}$ superconducting layered compounds. The conception of the intrinsic pinning associated with the periodic layered structure was formulated for vortices parallel to the layer planes $[33,35]$. High- $T_{c}$ compounds are usually considered as the periodic stacking of the strongly and weakly superconducting (or normal) layers [35]. The origin of the intrinsic pinning is connected with the fact that the most energetically favorable position of the vortex line, which is parallel to the layers, corresponds to its location in a weakly superconducting, either normal or insulating, interlayer. In this case, the gain in the vortex free energy is about equal to the condensation energy in the volume of the vortex cores. Moreover, the vortices are pinned along their entire length. When the distance between vortices in the direction orthogonal to the layers matches with the layered structure period, all the vortices are locked between superconducting layers. This configuration corresponds to the strong pinning. If the magnetic field and transport current both lie in the layer plane and orthogonal each to other, the vortices should move under the influence of the Lorentz force across the layers, i.e., overcome the high potential barriers associated with the variation of the condensation energy. Such a movement is strongly hindered and occurs by the finite steps at rather high currents [6]. The important role of the intrinsic pinning, determining the orientational dependence of the critical current $I_{c}$, was confirmed experimentally [36-38]. Intrinsic pinning also gives rise to such phenomenon as a lock-in transition [25].
Due to intrinsic pinning the essential modification of the vortex lattice structure in comparison with Abrikosov one is also expected. Recently, the influence of commensurability effect on the vortex arrangement in a parallel magnetic field has been considered in the theoretical works $[3-6,8,39]$. At a given magnetic field, one can make the VL be commensurate with the underlying layered structure by the compressing the VL along the normal to the layers and introducing the necessary shear deformation [4]. However, if the energy $E_{\mathrm{el}}$ of such a deformation exceeds the pinning energy $E_{p}$, the VL parameters are determined by the applied magnetic field. Then it is the value of the external magnetic field that would prescribe whether the lattice is commensurate or not with the underlying periodic pinning potential. It is a situation which should be characteristic for the weakly layered systems. This situation does not differ basically from that considered by Ami and Maki. However, in the case of comparatively large values of the anisotropy parameter $\gamma$ the final result for the «resonant» fields [4] turns out to be different from (1):

$$
H_{N}=\frac{\sqrt{3}}{2} \frac{\Phi_{0}}{N^{2} s^{2} \gamma} ; \quad \gamma=\left(\frac{M}{m}\right)^{1 / 2}
$$

Here $N=1,2,3 \ldots$ is the order of commensurability. Formula (2) corresponds to the simplest case of rhombic VL with one of its unit-cell vectors parallel to the layers. It is easy to see that formula (2) differs from the Ami-Maki expression for the case $L=0$ only by the factor $\gamma$, which is absent in the Ami-Maki expression for $H_{\Delta}$ because of very insignificant anisotropy incorporated in their model. The depinning current should oscillate, reaching maximal values at the integer $Z_{0} / s$ values. If other pinning centers are absent, the critical current should be zero at all $H$ values where the ratio $Z_{0} / s$ is not close to the integer numbers.

Intrinsic pinning energy increases with the lowering of temperature as at any other pinning mechanism. Moreover, as was mentioned above, it turns out more pronounced when the vortex core size in the direction orthogonal to the layers $\xi_{\perp}(T)$ becomes comparable to or smaller than the superstructure period. In this case the vortex cores fit easily between two neighboring superconducting layers. Taking into account the ratio between $\xi_{\perp}(T)$ and $s$, Ivlev, Kopnin, and Pokrovsky (IKP) [4] have classified all layered systems as superconductors with weak layering $\left(\xi_{\perp}(T)>>s\right)$ and strong layering $\left(\xi_{\perp}(T)<<s\right)$. According to IKP, the regime of strong pinning is accessible for any type of layering, and in both the cases of weak and strong layering there are regions of the $H-T$ diagram where the elastic deformation energy is smaller than the pinning energy [4]. For 
weakly layered superconductors $\left(\xi_{\perp}(T)>>s\right)$ such regions are determined by the conditions:

$$
\begin{aligned}
1-\frac{H}{H_{c 2}} & <\left(\frac{\xi_{c}}{s}\right)^{3} \exp \left(-\frac{8 \xi_{c}^{2}}{s^{2}}\right) ; \\
\frac{H}{H_{c 2}} & <\left(\frac{\xi_{c}}{s}\right)^{3} \exp \left(-\frac{8 \xi_{c}^{2}}{s^{2}}\right),
\end{aligned}
$$

while for highly layered structures $\left(\xi_{\perp}(T)<<s\right)$ they are determined by the conditions:

$$
1-\frac{H}{H_{c 2}}<1 ; \quad \frac{H}{H_{c 2}}<1 .
$$

The estimates by formula (3) show that for weakly layered superconductors the regions of the strong pinning are unrealistically narrow. Thus the more realistic statement is that for the layered systems with $\xi_{\perp}(T=0)>s$ the pinning is relatively weak at all $T$ and $H$. If $\xi_{\perp}(T=0)<s$, then at the elevated temperatures $\xi_{\perp}(T)$ may become $\geq s$, and the transition between the situation of strong and weak layering may occur. It is clear that in the frame of the above-mentioned classification the concentrationally modulated alloys and artificial superlattices of $\mathrm{S} / \mathrm{S}^{\prime}$ and $\mathrm{S} / \mathrm{N}$-type ( $\mathrm{S}$ is a superconductor, $\mathrm{S}^{\prime}$ is a superconductor with $T_{c}$ smaller than one in $\mathrm{S}, \mathrm{N}$ is a normal metal) should belong to the weakly layered systems. The superlattices of S/I-type (I is a semiconductor or an insulator) may belong either to the weakly or to the strongly layered systems. This depends on anisotropy parameter and, respectively, on the interlayer Josephson coupling energy. Both situations may be expected in high- $T_{c}$ superconductors. The authors of the works $[4,5]$ noted that $\mathrm{YBa}_{2} \mathrm{Cu}_{3} \mathrm{O}_{x}$, in particular, could be a favorable object for the investigation of the commensurability effects. Indeed, such experimental observations on $\mathrm{YBa}_{2} \mathrm{Cu}_{3} \mathrm{O}_{x}$ and on $\mathrm{RBa}_{2} \mathrm{Cu}_{3} \mathrm{O}_{x} \quad(\mathrm{R}=\mathrm{Y}, \mathrm{Nd})$ have appeared later [40-43]. In the work of Oussena et al. [40] the oscillations in the magnetization of $\mathrm{YBa}_{2} \mathrm{Cu}_{3} \mathrm{O}_{x}$ single crystal were observed when magnetic field was parallel to its $\mathbf{a}$ or $\mathbf{b}$ axis. The positions of the magnetization maxima were temperature independent. It was clear evidence that the oscillations are connected with the matching between vortex lattice parameter and regular layered structure. The large number of maxima on the magnetization curves (the magnetic field was varied up to $12 \mathrm{~T}$ ) allowed the authors to determine the anisotropy parameter in $\mathrm{YBa}_{2} \mathrm{Cu}_{3} \mathrm{O}_{x}$ to good accuracy using formula (2) for the case of weak layering. In particular, using the data of Fig. 3 of Ref. 40 (upper panel), presented for $\mathbf{H}$ parallel to the $\mathbf{a}$ axis and $T=60 \mathrm{~K}$, one can easily determine the orders of commensurability present on this plot. The maxima fit to the orders $N$ from 5 to 11 . The maxima corresponding to the smaller $N=1-4$ should be observed at $H>12 \mathrm{~T}$. Because of the relatively large wavelengths in our multilayers the $H_{N}$ values belonging to the low orders of commensurability get in the accessible range of magnetic fields in distinction to the high-temperature compounds with small $s$ values. At $60 \mathrm{~K}$ the additional peak on the magnetization oscillation curve is observed which does not obey the above-mentioned classification and was not explained by the authors. We will present the interpretation of this extra peak below. It is somewhat surprising that the $M$ oscillation amplitude diminishes at lower temperatures. Probably, it means that background pinning on another type of defects grows with decreasing $T$ faster than the intrinsic pinning. In the work of Gordeev et al. [41] the oscillations of resistance with parallel magnetic field have been observed on YBCO samples with the transition temperature $60 \mathrm{~K}$. The features of the resistive transitions on this sample are similar to those observed on our SLs.

As was mentioned above, in the situation of the strong layering and strong pinning, the VL should be commensurate with SL wavelength at all magnetic field values [4]. According to IKP, the stable states of the commensurate lattice correspond to the rhombic lattice with the rational values of the apex angle $\pi / 3$ and $2 \pi / 3$ (in the frame of the reduced coordinates $x, z \sqrt{M / m}$; here $x$ is the direction along the layers, and $z$ is along the $\mathbf{c}$ axis). The conditions of stability look as follows [4]:

$$
p=\pi / \sqrt{3}, \quad p=\pi \sqrt{3} .
$$

Here $p$ is the reduced magnetic field:

$$
p=2 \pi N^{2} s^{2} \gamma H / \Phi_{0} .
$$

Correspondingly, the dependence of the free energy for the given commensurate lattice on magnetic field has two minima at the $H$ values:

$$
H_{N}^{(1)}=\frac{\Phi_{0}}{2 \sqrt{3} N^{2} s^{2} \gamma}, \quad H_{N}^{(2)}=\frac{\Phi_{0} \sqrt{3}}{2 N^{2} s^{2} \gamma} .
$$

Note that the $H_{N}^{(2)}$ values coincide with $H_{N}$ for weakly layered structures. We have already mentioned above that the patterns of the resistance minima obtained in the different $R$ vs $H_{\|}$realizations have nothing in common with calculated values of the Ami-Maki fields $H_{\Delta}$. As follows from the above argumentation about the temperature range in which the resistance minima and ZRR states are observed on $\mathrm{Mo} / \mathrm{Si}$ samples investigated, for both $\mathrm{Mo} / \mathrm{Si}$ and $\mathrm{W} / \mathrm{Si}$ SLs we are dealing with the case of highly layered superconductors. That is why the comparison 


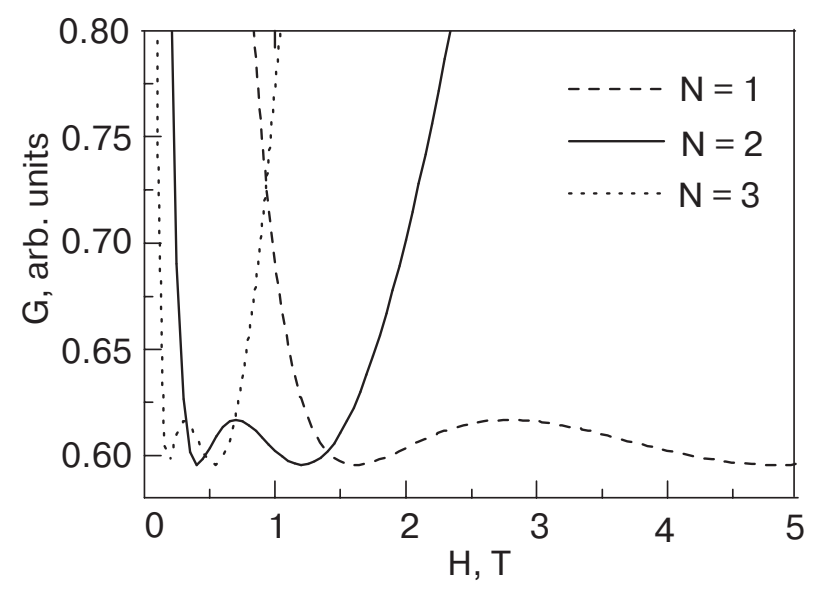

Fig. 9. Dependences of the Gibbs free energy on magnetic field for different orders of commensurability for $\mathrm{Mo} / \mathrm{Si}$ sample B calculated according to IKP theory.

with IKP theory considering this limiting case is the most reasonable. Figure 9 shows the dependences of the Gibbs energy on magnetic field calculated according to IKP for different orders of commensurability for $\mathrm{Mo} / \mathrm{Si}$ sample B, with the $\gamma$ value of 11.8. A comparison of the pattern of Fig. 9 with the data obtained on this sample reveals quite satisfactory consistency with the IKP theoretical predictions. In Fig. 3 the two regions of the ZRR states correspond to the two stable states for $N=1$. The $H_{N}$ values are shown in this figure by the thick arrows. Minima in Fig. 4 correspond to $N=3$, the second stable state, and $N=1$, the first stable state. The stable state with $N=2$ is also obtained in one of the realizations. All the $R$ vs $H_{\|}$realizations observed correspond to the definite initial conditions under which the vortex lattice was formed. As was mentioned above, the realization shown in Fig. 3 is observed in a case when the external magnetic field is switched on first at sufficiently low temperature. The first ZRR state in this case corresponds to $N=1$. It is obvious that in such a case the appearance of the states with the larger $N$ is impossible in the increasing field. When the measurements of the resistive curves are started from $T_{c}$, the realizations of such types as presented in Figs. 1 and 4 take place. The positions of the IKP fields $H_{N}$ in these figures are also indicated by the thick arrows.

For Mo/Si sample A, with smaller $s$ and $\gamma$ values, we have the following values of the IKP matching field at two stable states: for $N=1,4.2 \mathrm{~T}$ and $12,6 \mathrm{~T}$; for $N=2,1.05 \mathrm{~T}$ and $3.15 \mathrm{~T}$; for $N=3,0.47 \mathrm{~T}$ and $1.4 \mathrm{~T}$. Comparison of these values with Fig. 2 shows that the $R$ minima corresponding to $N=3$ and $N=2$ (for both minima it is the second stable state) are distinctively seen. They are indicated by the thick arrows.

Between these two minima there is additional minimum at a field of $2.02 \mathrm{~T}$ (it is shown in Fig. 2 by thin arrow), which fairly well corresponds to the IKP stable state with a half-integer order of commensurability $N=2.5$. From our point of view, such commensurate arrangements are also allowed in the strongly layered superconductors. One should simply contemplate the possibility that another kind of commensurate states exists, when half of the vortex chains are confined to the spacers while the other half are located on the superconducting layers. It is a regular vortex lattice consisting of the Josephson and Abrikosov vortices. The additional evidence for the existence of such VLs follows from data obtained on $\mathrm{Mo} / \mathrm{Si}$ SLs with thick spacers, where only magnetic interlayer coupling is essential, and avalanche-type jumps of resistance correspond to phase transitions between different commensurate states [44]. It is known that a single flux line sitting on a superconducting layer is unstable [45]. However, in a case when half of the vortices are trapped in the spacers and fixed by the strong periodic pinning potential, the entire vortex system is commensurate with this potential and stable. A similar observation in commensurability magnetization experiments on high- $T_{c}$ samples was reported in Ref. 43. This situation is also reminiscent of one which occurs in the case of a superconducting film with an antidote lattice, where in the matching field all of the vortex ensemble is strongly pinned, though only part of vortices are trapped at pinning sites [46].

Two observed $R$ minima on sample $\mathrm{C}$ may be identified as IKP stable states for $N=1$ and $N=3$ [28]. Sometimes, as particularly in the latter case and the case illustrated by Fig. 4 for sample B, the stable state with $N=2$ is not observed experimentally. The possible explanation of this fact might be as follows. The rearrangement of the VL at the transition from the lattice with $N=3$ to the lattice with $N=2$ is rather complicated, while the transition $N=3 \rightarrow N=1$ occurs simply by the dividing of one vortex chain into three. Nevertheless, this transition is observed on sample A.

A fairly good consistency with the IKP theory is obtained on $\mathrm{W} / \mathrm{Si}$ samples as well. On the sample with $s=50 \AA$ the first two $R$ minima correspond to $N=4$ and $N=2$, the first stable state (Fig. 7). The third minimum fits as in a case with $\mathrm{Mo} / \mathrm{Si}$ sample A to a half-integer number 1.5 . On $\mathrm{W} / \mathrm{Si}$ sample with $s=60 \AA$ the $R$ minima corresponding to two stable states with $N=4$ are found. The minimum corresponding to $N=3$, which should appear between these two minima, does not reveal itself. The reason for this might be the above-mentioned difficulty with the rearranging of the VL in the situation when two $N$ values are not multiples of each other.

As an additional confirmation that the resistive transition patterns reflect the properties of the com- 


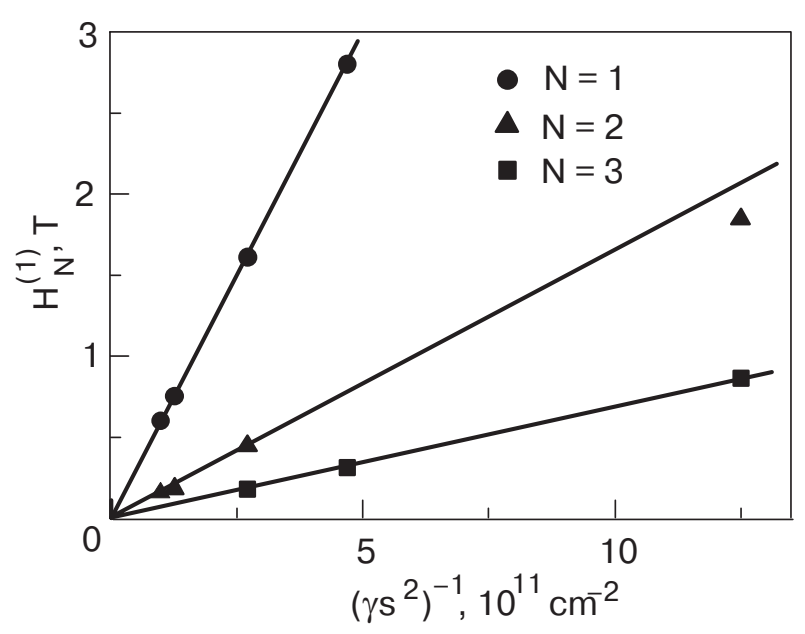

Fig. 10. Experimental values of the commensurate magnetic fields for samples with different wavelengths and anisotropy parameters.

mensurate lattices may serve such data as ones presented in Fig. 10. Here the examples of the dependence of experimental values of $H_{N}$ on the parameter $\left(\gamma s^{2}\right)^{-1}$ characterizing the individuality of the layered sample are shown. As follows from formula (7), the data for different samples lie on straight lines passing through the zero point. Thus by changing the spacer thickness at a constant metal layer thickness, one obtains additional evidence of the perfect applicability of the IKP theory for the interpretation of the oscillation phenomena and ZRR states.

Analysis of the data of Oussena et al. [40] has shown that the extra peak in magnetization observed on YBCO at the relatively low temperature $60 \mathrm{~K}$ is the peak corresponding to IKP field for a strongly layered system with $N=9$ (the second stable state). Thus, the second stable states begin to appear at lower temperatures.

Besides the stable VL configurations considered by IKP, rather different VL states might exist, as was mentioned above, which are dynamically accessible at the variation of magnetic field $[4,6,7]$. Under conditions of strong pinning and relatively small magnetic fields, shear instability of the commensurate lattice leads to the breaking of symmetry: neighboring vortex chains locked between superconducting layers may be shifted with respect to one another [6,7]. A lot of these «shifted» lattices correspond to metastable states, i.e., to local minima of the free energy. The positions of all the free energy local minima have a hierarchical structure, which is described by a set of Farey numbers [7]. Due to this hierarchy the number of metastable states grows with magnetic field decrease. The trajectory of the dynamically accessible minimum, which starts from the symmetric VL (the lattice without shear), goes through a sequence of bifurcation and quasibifurcation points, and this trajectory is not uniquely defined. When magnetic field varies, the trace of the absolute minimum jumps in a complicated way between different metastable states [7]. One may believe that metastable states of the VL may be probed in dynamic experiments, i.e., by resistive or critical-current measurements. We suggest that a number of additional features observed on the $R$ vs $H$ and $I_{c}$ vs $H$ curves (like steps, kinks, or abrupt changes of curvature; see, for example, the thin arrows in Fig. 3) along with the main maxima and minima, may be evidence of a manifestation of dynamically accessible metastable states. Sometimes the $H$ values where the features mentioned appear are the same in different experiments, and sometimes these values change, i.e., other trajectories of dynamically accessible minima are realized.

An interesting aspect of the oscillation phenomena observed on $\mathrm{Mo} / \mathrm{Si}$ and $\mathrm{W} / \mathrm{Si}$ multilayers is a memory effect. It manifests itself in the repetition of the same $R\left(H_{\|}\right)$curve patterns during all experiments made in the same run of the cooling down of the sample. If one wishes to obtain another realization of the resistive behavior, it is necessary to heat the sample above the transition temperature. The memory effect has been observed on all samples investigated. Subsequently to the diminishing of the magnetic field down to zero, part of the vortices stay frozen in all interlayers where they were during the previous field sweep. Evidence of that is the hysteretic behavior. This predetermines the appearance of the same resistive pattern at each following field sweep at lower temperatures. It seems that during the decrease of the field and, correspondingly, of the vortex density, the VL might pass through another sequence of transformations. For example, if in the increasing field $H_{i}$ one observes a transition between commensurate VL phases with $N=3$ and $N=1$, nothing should preclude, it seems, the transition $N=1 \rightarrow N=2$ in the decreasing field $H_{d}$. Nevertheless, this never happens, and the resistive curves in field $H_{d}$ repeat the pattern observed in field $H_{i}$. Therefore one arrives at the conclusion that the previous location of the vortex rows in definite interlayers modifies in some way the intrinsic pinning behavior. To eliminate this subtle modification the sample should be returned to the virgin state, i.e., be put in the normal state. It is noteworthy that the memory effect concerns the metastable states as well (see, for example, Figs. 3 and 6). For a full understanding of the memory effect, additional experiments are needed.

Attention must also be paid to the fact that the set of the different $R\left(H_{\|}\right)$realizations on each sample constitutes a unique «fingerprint», which characterizes the possible stable VL states for a given multilayer. This 
fingerprint depends exclusively on the anisotropy parameter and SL period (more exactly, on the value $\gamma s^{2}$ ), and does not depend on the type of materials used for preparation of the multilayer. Obviously, it is possible to imagine the eventuality of the existence of the «twin» samples with the different values of $\gamma$ and $s$, but with equal $\gamma s^{2}$ magnitude. However, as a matter of fact, such coincidence is highly improbable due to the very strong dependence of the anisotropy parameter on the spacer thickness [29]. Thus, the investigations of the commensurate states in the layered superconductors may be used for determination of the anisotropy parameter $\gamma$. The same conclusion follows from the magnetization experiments of Oussena et al. However, it is better to deal with samples having a rather large number of layers because in the layered thin films commensurability with the full sample thickness is possible as well [47]. The interplay between the two commensurability effects leads to the appearance of special selection rules. This makes the oscillation data interpretation more complicated.

The predictions about VL structure in layered superconductors obtained in the paper of Bulaevskii and Clem (BC) [8] in the framework of the LD model, which takes into account the Josephson nature of the interlayer coupling, are rather different from those obtained on the base of 3D anisotropic London or GL approach. The series of the first-order phase transitions between different commensurate phases should occur at definite magnetic field values $H_{n, n-1}$, which also depend on the anisotropy parameter and SL wavelength. For this effect the value of the characteristic field $H_{0}=\Phi_{0} / \gamma s^{2}$ where Josephson cores of the vortices begin to overlap is essential.

For sample B this characteristic field $H_{0}$ is equal to $5.51 \mathrm{~T}$. The transition between commensurate phases corresponding to $Z_{0}=s$ and $Z_{0}=2 s$ should occur in the field $H_{2,1}=H_{0} / 3=1.84 \mathrm{~T}$, and another transition (between the phases with $Z_{0}=2 s$ and $Z_{0}=3 s$ ) in the field $H_{3,2}=H_{0} / 8=0.69 \mathrm{~T}$. The position of the large minimum in the Fig. 1 is just consistent with the field $H_{2,1}$ estimated above on the base of LD model results of $\mathrm{BC}$ [8]. However, at fields close to $H_{3,2}=0.69 \mathrm{~T}$ there is only a peculiarity on the derivative $d R / d H$ (data of Fig. 1,a). The second shallow minimum in Fig. 5, showing another run under the same conditions for sample B, is closer to the IKP field $H_{3}^{(2)}$.

It is necessary to note here that the values of IKP and $\mathrm{BC}$ fields are rather close to each other numerically. The ratio of the fields $H_{0} / H_{N}^{(2)}=2 N^{2} / \sqrt{3} \approx 1$ in the case $N=1$ and the ratio $H_{2,1} / H_{N}^{(1)}$ are equal to the same value. It is quite natural that the $\mathrm{BC}$ transition fields may be seen in the same magnetic field range as the IKP stable commensurate states. In the
IKP work the properties and stability of a given commensurate state are considered, but transitions between different commensurate states are outside the framework of this theory. Oppositely, in BC theory only the transitions between different commensurate VL states are under consideration. The approaches used in both papers mentioned are rather distinct, but the authors of these works are actually considering different sides of the same phenomenon, i.e., evolution of the mixed state in strongly layered superconductors. It is clear that at the appropriate choice of the current the both IKP and BC scenarios may be observed on the same sample. It appears that the location of the minima in Fig. 1, $a$ cannot be explained in the IKP GL model, while they are in excellent agreement with the results of the LD model of BC. In the meantime, the data of the other figures with the oscillating $R$ vs $H$ dependences are consistent only with the IKP theory predictions.

In conclusion, the new type of reentrance phenomenon discovered on Mo/Si multilayers, occurring if the magnetic field is aligned along the layers or slightly inclined with respect to the layer planes, has been studied in detail on series of $\mathrm{Mo} / \mathrm{Si}$ and $\mathrm{W} / \mathrm{Si}$ multilayered samples. The reentrance behavior is connected with the intrinsic pinning influence and with the specifics of the VL structure in layered superconductors. Locations of the $R$ vs $H$ minima and ZRR correspond either to the stable states of commensurate VL or to the transitions between two commensurate lattices with different $Z_{0}$. Examination of the results obtained allows one to conclude that investigation of the reentrance behavior may be used as a new tool for studying the VL arrangement in layered superconductors. The investigations of the dependences of the critical current on $H_{\|}$and of the resistive transitions give practically the same results. However, the latter method seems more attractive because it is essentially simpler, and it allows one to cover a larger area of the $H_{\|}-T$ diagram.

It has been shown experimentally that the state of the vortex matter in layered superconductors differs essentially from that in type-II superconductors with a random distribution of pinning centers.

\section{Acknowledgements}

This research was supported in part by Grant N 351/99 from the Israel Science Foundation and «The Center for Absorption in Science», Ministry of Immigrant Absorption, State of Israel. The authors are grateful to L. Bulaevskii, V. Kogan, and V.L. Pokrovsky for valuable discussions and to S.A. Yulin and R. Senderak for assistance in sample preparation. 
1. S. Ami and K. Maki, Progr. Theor. Phys. 53, 1 (1975).

2. H. Raffy, J.C. Renard, and E. Guyon, Solid State Commun. 11, 1679 (1972).

3. L.J. Campbell, M.M. Doria, and V.G. Kogan, Phys. Rev. B38, 2439 (1988).

4. B.I. Ivlev, N.B. Kopnin, and V.L. Pokrovsky, J. Low Temp. Phys. 80, 187 (1990).

5. B.I. Ivlev and N.B. Kopnin, Phys. Rev. Lett. 64, 1828 (1990).

6. B.I. Ivlev and N.B. Kopnin, J. Low Temp. Phys. 80, 161 (1990).

7. L.S. Levitov, Phys. Rev. Lett. 66, 224 (1991).

8. L. Bulaevskii and J.R. Clem, Phys. Rev. B44, 10234 (1991).

9. M. Ichioka, Phys. Rev. B51, 9423 (1995).

10. D.A. Huse, Phys. Rev. B46, 8621 (1992).

11. L.N. Bulaevskii, M. Ledvij, and V.G. Kogan, Phys. Rev. B46, 366 (1992).

12. A. Sudbø, E.H. Brandt, and D.A. Huse, Phys. Rev. Lett. 71, 1451 (1993).

13. L.L. Daemen, L.J. Campbell, A.Yu. Simonov, and V.G. Kogan, Phys. Rev. Lett. 70, 2948 (1993).

14. G. Preosti and P. Muzikar, Phys. Rev. B48, 9921 (1993).

15. E. Sardella and M.A. Moore, Phys. Rev. B48, 9664 (1993).

16. A.V. Balatskii, L.I. Burlachkov, and L.P. Gor'kov, Zh. Eksp Teor. Fiz. 90, 1478 (1986) [Sov. Phys. JETP 63, 866 (1986)].

17. A.I. Buzdin and A.Yu. Simonov, Pis'ma Zh. Eksp. Teor. Fiz. 51, 168 (1990) [JETP Lett. 51, 191 (1990)].

18. A.E. Koshelev, Phys. Rev. Lett. 83, 187 (1999).

19. A.N. Grigorenko, S.J. Bending, A.E. Koshelev, J.R. Clem, T. Tamegai, and S. Ooi, Phys. Rev. Lett. 89, 217003 (2002).

20. P.H. Kes, J. Aarts, V.M. Vinokur, and C.J. van der Beek, Phys. Rev. Lett. 64, 1063 (1990).

21. M. Benkraouda and M. Ledvij, Phys. Rev. B51, 6123 (1995).

22. C.A. Bolle, P.L. Gammel, D.G. Grier, C.A. Murray, and D.J. Bishop, Phys. Rev. Lett. 66, 112 (1991).

23. I.V. Grigorieva, J.W. Steeds, and K. Sasaki, Phys. Rev. B48, 16865 (1993).

24. G.J. Dolan, F. Holtzberg, C. Feild, and T.R. Dinger, Phys. Rev. Lett. 62, 2184 (1989).

25. D. Feinberg and C. Villard, Phys, Rev. Lett. 65, 919 (1990).

26. A.M. Grishin, A.Yu. Martynovich, and S.V. Yampol'skii, Zh. Eksp. Teor. Fiz. 97, 1930 (1990) [Sov. Phys. JETP 70, 1089 (1990)].

27. C. Coccorese, C. Attanasio, L.V. Mercaldo, M. Salvato, L. Maritato, J.M. Slaughter, C.M. Falco, S.L. Prischepa, and B.I. Ivlev, Phys. Rev. B57, 7922 (1998).
28. N.Ya. Fogel, M.Yu. Mikhailov, Yu.V. Bomze, and O.I. Yuzephovich, Phys. Rev. B59, 3365 (1998).

29. M.Yu. Mikhailov, O.I. Yuzephovich, A.S. Pokhila, Yu.V. Bomze, N.Ya. Fogel, I.M. Dmitrenko, S.A. Yulin, A.S. Sidorenko, O.B. Moldovan, and E.I. Buchstab, Fiz. Nizk. Temp. 25, 850 (1999) [Low Temp. Phys. 25, 635 (1999)].

30. E.I. Bukhshtab, V.Yu. Kashirin, V.V. Kondratenko, A.I. Fedorenko, N.Ya. Fogel, V.G. Cherkasova, and S.A. Yulin, Fiz. Nizk. Temp. 19, 704 (1993) [Low Temp. Phys. 19, 506 (1993)].

31. N.Ya. Fogel, E.I. Buchstab, A.S. Pokhila, A.I. Erenburg, and V. Langer, Phys. Rev. B53, 71 (1996).

32. O.I. Yuzephovich, Yu.V. Bomze, M.Yu. Mikhailov, I.M. Dmitrenko, and N.Ya. Fogel, Fiz. Nizk. Temp. 26, 142 (2000) [Low Temp. Phys. 26, 103 (2000)].

33. M. Tachiki and S. Takahashi, Solid State Commun. 70, 291 (1989).

34. P.R. Broussard and T.H. Geballe, Phys. Rev. B37, 68 (1988).

35. M. Tachiki and S. Takahachi, Solid State Commun. 72, 1083 (1989).

36. B. Roas, L. Schultz, and G. Saemann-Ischenko, Phys. Rev. Lett. 64, 479 (1990).

37. P. Schmitt, P. Kummeth, L. Schultz, and G. Saemann-Ischenko, Phys. Rev. Lett. 67, 267 (1991).

38. A. Walkenhorst, C. Tome-Rosa, C. Stolzel, G. Jacob, M. Schmitt, and H. Adrian, Physica C177, 165 (1991).

39. V. Prokic, D. Davidovic, and L. Dobrosavljevic-Grujic, Phys. Rev. B51, 6013 (1995).

40. M. Oussena, P.A.J. de Groot, R. Gagnon, and L. Taillefer, Phys. Rev. Lett. 72, 606 (1994).

41. S.N. Gordeev, A.A. Zhukov, P.A.J. de Groot, A.G.M. Jansen, R. Gagnon, and L. Taillefer, Phys. Rev. Lett. 85, 4594 (2000).

42. H. Kupfer, G. Ravikumar, T. Wolf, A.A. Zhukov, A. Will, H. Leibrock, R. Mtetr-Himer, H. Wuhl, and P.A.J. de Groot, Phys. Rev. B66, 64512 (2002).

43. H. Kupfer, G. Ravikumar, A.A. Zhukov, P.A.J. de Groot, C. Meingast, A.I. Rykov, S. Tajima, and Th. Wolf, Physica C404, 215 (2004).

44. O.I. Yuzephovich, Yu.V. Bomze, M.Yu. Mikhailov, E.I. Buchstab, and N.Ya. Fogel, Physica C361, 59 (2001).

45. T. Koyama, N. Takezawa, and M. Tachiki, Physica C172, 501 (1991).

46. V.V. Metlushko, L.E. DeLong, M. Baert, E. Rosseel, M.J. van Bael, K. Temst, and V.V. Moshchalkov, Europhys. Lett. 41, 333 (1998).

47. N.Ya. Fogel, E.I. Buchstab, V.G. Cherkasova, O.I. Yuzephovich, M.Yu. Mikhailov, and A.N. Stetzenko, Fiz. Nizk. Temp. 27, 1019 (2001) [Low Temp. Phys. 27, 752 (2001)]. 